\title{
Automatic Classification of Containment and Support Spatial Relations in English and Dutch
}

\author{
Kate Lockwood, Andrew Lovett, and Ken Forbus \\ Qualitative Reasoning Group, Northwestern University \\ 2133 Sheridan Rd \\ Evanston, IL 60208 \\ \{kate, andrew-lovett, forbus\}@northwestern.edu
}

\begin{abstract}
The need to communicate and reason about space is pervasive in human cognition. Consequently, most languages develop specialized terms for describing relationships between objects in space - spatial prepositions. However, the specific set of prepositions and the delineations between them vary widely. For example, in English containment relationships are categorized as in and support relationships are classified as on. In Dutch, on the other hand, three different prepositions are used to distinguish between different types of support relations: op, aan, and om. In this paper we show how progressive alignment can be used to model the formation of spatial language categories along the containment-support continuum in both English and Dutch.
\end{abstract}

Keywords: Cognitive modeling, spatial prepositions

\section{Introduction}

Being able to reason and communicate about space is important in many human tasks from hunting and gathering to engineering design. Virtually all languages have developed specialized terms to describe spatial relationships between objects in their environments. In particular, we are interested in spatial prepositions. Spatial prepositions are typically a closed-class of words and usually make up a relatively small part of a language. For example, in English there are only around 100 spatial prepositions. Understanding how people assign spatial prepositions to arrangements of objects in the environment is an interesting problem for cognitive science.

Several different aspects of a scene have been shown to contribute to spatial preposition assignment: geometric arrangement of objects, typical functional roles of objects (e.g. [9]), whether those functional relationships are being fulfilled (e.g. [4]) and even the qualitative physics of the situation (e.g. [5]). The particular elements that contribute to prepositions and how they are used to divide the space of prepositions has been found to vary widely between languages (e.g. [1, 2]).

This paper shows how progressive alignment can be used to model how spatial prepositions are learned. Progressive alignment uses the structural alignment process of structure-mapping theory to construct generalizations from an incremental stream 
of examples. The specific phenomena we model here is how people make distinctions along the containment-support continuum in both English and Dutch, based on a psychological experiment by Gentner and Bowerman [11]. To reduce tailorability in encoding the stimuli, we use hand-drawn sketches which are processed by a sketch understanding system. We show that our model can learn to distinguish these prepositions, using (as people do) semantic knowledge as well as geometric information, and requiring orders of magnitude fewer examples than other models of learning spatial prepositions.

The next section describes the Gentner and Bowerman study that provided the inspiration for our experiments. Section 3 reviews structure-mapping theory, progressive alignment, and the analogical processing simulations we use in our model. It also summarizes the relevant aspects of CogSketch, the sketch understanding system we used to encode the stimuli, and the ResearchCyc knowledge base we use for common sense knowledge. Section 4 describes the simulation study. We conclude by discussing related work, broader issues, and future work.

\section{Gentner and Bowerman's study of English/Dutch prepositions}

Gentner and Bowerman [11] were testing the Typological Prevalence hypothesis that the frequency with which distinctions and categories are found across the world's languages provides a clue to conceptual "naturalness" and how easy that particular distinction is to learn. To explore this, they focused on a subset of spatial prepositions in English and Dutch. The English and Dutch languages divide the supportcontainment continuum quite differently. In English there are two prepositions: in is used for containment relationships and on is used for support relationships. However, Dutch distinguishes three different forms of support. The prepositions for Dutch and English are outlined in Table 1 below.

Bowerman and Pederson found in a previous study [1] that some ways of dividing up the containment-support continuum are very common crosslinguistically while others are relatively rare. English follows a more linguistically common approach by grouping all support relations together into the on category while the Dutch op-omaan distinction is extremely rare. Both use the very common containment category. Following the Typological Prevalence Hypothesis, both English and Dutch children should learn the common and shared category of in around the same time. It should take Dutch children longer to learn the rare aan/op/om distinctions for support than it takes the English children to learn the common on category. 
Table 1. Table showing the containment and support prepositions in English and Dutch. Drawings here are taken from the original Genter and Bowerman paper.

\begin{tabular}{|c|c|l|l|}
\hline English & Dutch & Relationship & Example \\
\hline on & op & support from below & hanging attachment \\
\hline on & aan & om & encirclement with contact \\
\hline on & in & containment \\
\hline in & om \\
\hline
\end{tabular}

\subsection{Experiment}

They tested children in five age groups (2, 3, 4, 5, and 6 years old) as well as adults who were native speakers of English and Dutch. Each subject was shown a particular arrangement of objects and asked to describe the relationship in their native language. In the original experiment, 3-dimensional objects where used. So, for example, a subject would be shown a mirror on the wall of a doll house and asked "Where is the mirror". The set of all stimuli is shown in Table 2 below.

Table 2. Stimuli from the Gentner and Bowerman study

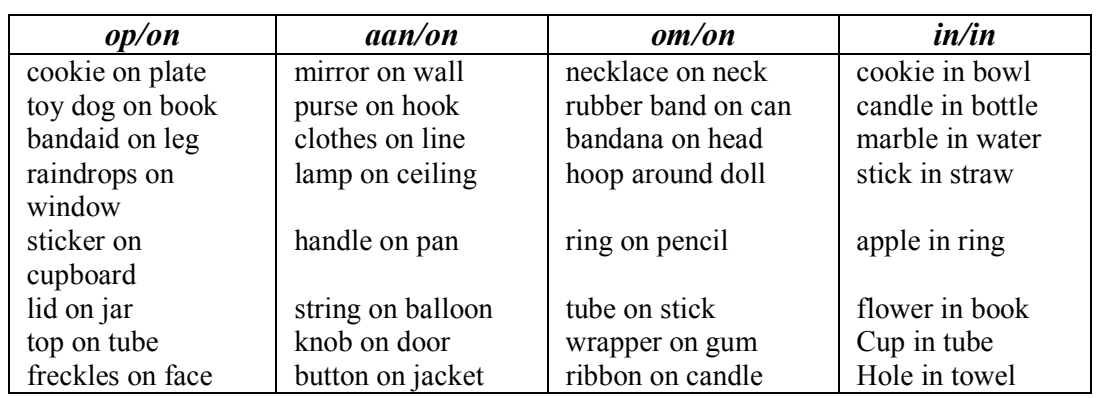

The results of the study were consistent with the Typological Prevalence hypothesis. Specifically, Dutch children are slower to acquire the op, aan, om system of support relations than English children are to learn the single on category. Both groups of children learned the in category early and did not differ in their proficiency using the term. Across all prepositions, English-speaking 3 to 4 year old children 
used the correct preposition $77 \%$ of the time, while the Dutch children used the correct preposition $43 \%$ of the time. Within the Dutch children, the more typical op category was learned sooner than the rarer aan and om categories. For a more detailed description of the results, please see the original paper.

\subsection{Motivation for our simulation study}

Modeling these results in detail is a daunting challenge for cognitive simulation. To accurately capture the developmental trajectories of learning over multiple years, for example, requires constructing a body of stimuli whose statistical properties are based on hypotheses about the commonalities of experiences in the world. No cognitive simulation has ever operated at that scale. There are practical challenges as well as methodological challenges: automatic encoding of stimuli becomes essential, for instance, whereas most cognitive simulations operate over hand-coded representations. Consequently, in this paper we focus on a simpler, but still difficult, question: Can progressive alignment be used to learn the spatial language containment/support categories in both English and Dutch? We use the Gentner \& Bowerman stimuli as a starting point, a known set of good examples for each of these categories.

\section{Simulation Background}

Several existing systems were used in our simulation. Each is described briefly here.

\subsection{Simulating similarity via analogical matching}

We use Gentner's structure-mapping theory of analogy and similarity [12]. In structure-mapping, analogy and similarity are defined in terms of a structural alignment process operating over structured, relational representations. Our simulation of comparison for finding similarity is the Structure-Mapping Engine [8], which is based on structure-mapping theory. SME takes as input two cases, a base and a target. It produces as output between one and three mappings describing the comparison between base and target. Each mapping consists of: (1) correspondences between elements in the base and elements in the target; (2) a structural evaluation score, a numerical characterization of how similar the base and target are; and (3) candidate inferences, conjectures about the target made by projecting partially-mapped base structures. There is considerable psychological evidence supporting structure-mapping theory, including modeling visual similarity and differences $[13,17]$ and SME has been used to successfully model a variety of psychological phenomena.

\subsection{Progressive Alignment and SEQL}

Progressive alignment constructs generalizations by incremental comparisons, assimilating examples that are sufficiently similar into generalizations. These generali- 
zations are still rather concrete, and do not contain variables. Attributes and relationships that are not common "wear away", leaving the important commonalities in the concepts. Probabilities are associated with each statement in the generalization, which provides a way of identifying what aspects of the description are more common (and hence more central) to the generalization.

We model progressive alignment via SEQL $[14,15,20]$, which uses SME as a component. SEQL creates generalizations from an incoming stream of examples. A generalization context consists of a set of generalizations and examples for a concept. For example, in learning spatial prepositions, there would be one generalization context per preposition. All scenes described with the word $o p$, for example, would be processed in the $o p$ context. There can be more than one generalization per context, since real-world concepts are often messy and hence disjunctive.

When a new example arrives, it is compared against every generalization in turn, using SME. If it is sufficiently close to one of them (as determined by the assimilation threshold), it is assimilated into that generalization. The probabilities associated with statements that match the example are updated, and the statements of the example that do not match the generalization are incorporated, but with a probability of $1 / n$, where $n$ is the number of examples in that generalization. If the example is not sufficiently close to any generalization, it is then compared against the list of unassimilated examples in that context. If the similarity is over the assimilation threshold, the two examples are used to construct a new generalization, by the same process. An example that is determined not to be sufficiently similar to either an existing generalization or unassimilated example is maintained as a separate example.

\subsection{CogSketch}

CogSketch $^{1}$ is an open-domain sketch understanding system. Each object in a CogSketch sketch is a glyph. Glyphs have ink and content. The ink consists of polylines, i.e., lists of points representing what the user drew. The content is a symbolic token used to represent what the glyph denotes. In CogSketch, users indicate the type of the content of the glyph in terms of concepts in an underlying knowledge base. This is one form of conceptual labeling. The knowledge base used for this work is a subset of the ResearchCyc KB, which contains over 30,000 concepts. In addition to conceptual labels, the contents of glyphs can also be given names. A name is a natural language string that the user can use to refer to the content of the glyph.

CogSketch automatically computes a number of qualitative spatial relations and attributes for glyphs in a sketch. The relations computed include the RCC-8 qualitative relations [3] that describe all possible topological relations between two-dimensional shapes (e.g. disconnected, edge-connected, partially-overlapping). RCC-8 relations are also used to guide the computation of additional spatial relationships such as positional relations like right/left. CogSketch also computes two types of glyph groups: connected glyph groups and contained glyph groups. Connected glyph groups consist

11 Available online at http://spatiallearning.org/projects/cogsketch index.html. The publicly available version of $\mathrm{CogSketch}$ comes bundled with the OpenCyc $\mathrm{KB}$ as opposed to the ResearchCyc KB which was used for this work. 
of a set of glyphs whose ink strokes intersect. A contained glyph group consists of a single container glyph and all of the glyphs fully contained within it.

\subsection{ResearchCyc}

Consider the sketch below showing the stimuli "freckles on face". If you just look at the topological relationship between the freckle glyphs and the face glyph, they clearly form a contained glyph group with the face as the container and the freckles as the insider. As work by Coventry and others has shown [6], geometric properties are not sufficient to account for the way people label situations with spatial prepositions. A purely geometric account would declare freckles to be in the face, but we actually say freckles are on/op faces. To model such findings, we must use real-world knowledge as part of our simulation. For example, we know that freckles are physically part of a face. We use knowledge from the ResearchCyc ${ }^{2}$ as an approximation for such knowledge. Freckles, for example, are a subclass of PhysiologicalFeatureofSurface, providing the semantic knowledge that, combined with geometric information, enables us to model spatial preposition judgments. As the world's largest and most complete general knowledge base, ResearchCyc contains much of the functional information needed about the figure and ground objects in our stimuli.

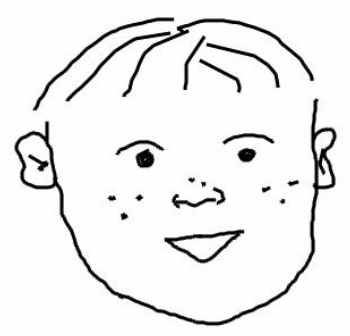

Fig. 1. Sketch of the spatial arrangement "freckles on face". If you examine just the geometric information, the freckles are in the area delineated by the face

\section{Experiment}

\subsection{Materials}

All 32 original stimuli from the Gentner and Bowerman study were sketched using CogSketch. Each sketch was stored as a case containing: (1) the automatically computed qualitative spatial relationships and (2) information about the types of objects in the sketch. In the original experiment subjects were cued as to which object should be the figure (e.g. "where is the mirror") and which should be the ground. To ap-

${ }^{2}$ http://research.cyc.com/ 
proximate this, each sketch contained two glyphs, one named figure and one named ground, and these names were used by the model. Recall that names in CogSketch are just strings that are used to refer to the objects. Each object was also conceptually labeled using concepts from the ResearchCyc KB. For instance, in the mirror on the wall stimulus, the mirror was declared to be an instance of the concept Mirror and the wall was labeled as an instance of Wall InAConstruction.

When people learn to identify spatial language categories in their native languages, they learn to focus on the relationships between objects, and to retain only the important features of the objects themselves rather than focusing on the surface features of the objects. As noted above, having conceptual labels and a knowledge base allows us to simulate this type of knowledge. For each conceptual label, additional concepts from its genls hierarchy were extracted from ResearchCyc. The genls hierarchy specifies subclass/superclass relationships between all the concepts of the KB. So, for example, Animal and Dog would both be genls of Daschund. Here we were particularly interested in facts relating to whether objects were surfaces or containers - and this was particularly important for ground glyphs. The original facts were removed (in our example "Daschund" would be deleted) to simulate abstraction away from specific object types to more important semantic categories.

In the original study, the physical objects used as stimuli were manipulated to make the important relationships more salient to subjects. We approximated this by drawing our sketches so as to highlight the important relationships for the individual spatial language categories. For example, the sketches for aan that required showing a connection by fixed points were drawn from an angle that made the connectivity between the parts observable. Figure 2 below shows two aan sketches: knob aan door and clothes aan line. They are drawn from perspectives that allow the system easy access to the point-contact relationship.

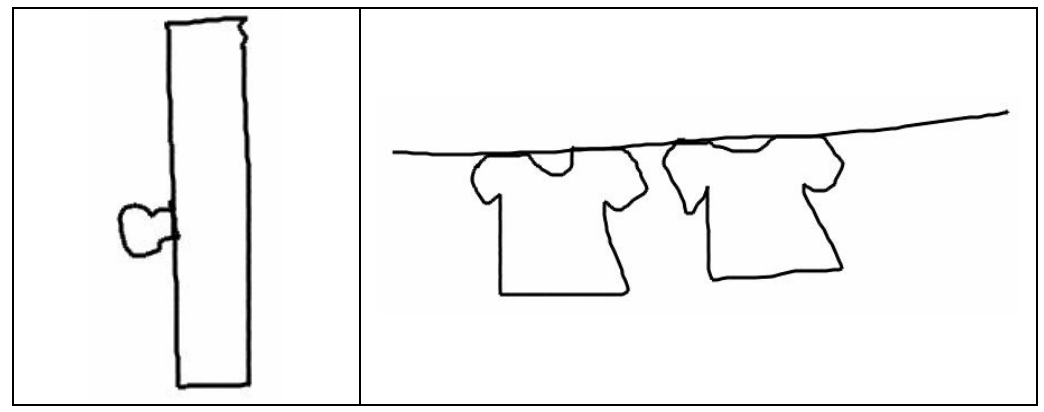

Fig. 2. Two sketched stimuli showing objects drawn from different angles to make the point connections salient

\subsection{Method}

The basic spatial category learning algorithm is this: For each word to be learned, a generalization context is created. Each stimulus representing an example of that word in use is added to the appropriate generalization contexts using SEQL. (Since we are 
looking at both Dutch and English, each example will be added to two generalization contexts, one for the appropriate word in each language.) Recall that SEQL can construct more than one generalization, and can include unassimilated examples in its representation of a category.

We model the act of assigning a spatial preposition to a new example $\mathrm{E}$ as follows. We let the score of a generalization context be the maximum score obtained by using SME to compare E to all of the generalizations and unassimilated examples in that context. The word associated with the highest-scoring generalization context represents the model's decision.

To test this model, we did a series of trials. Each trial consisted of selecting one stimulus as the test probe, and using the rest to learn the words. The test probe was then labeled as per the procedure above. The trial was correct if the model generated the intended label for that stimulus. There were a total of 32 trials in English ( 8 for in and 24 for on) and 32 trials in Dutch (8 each for in, op, aan, and om) one for each stimulus sketch.

\subsection{Results}

The results of our experiment are shown below. The generalizations and numbers given are for running SEQL on all the sketches for a category. The table below summarizes the number of sketches that were classified correctly, for each preposition the number is out of 8 total sketches except for English on which has 24 total sketches. All results are statistically significant $\left(\mathrm{P}<10^{-4}\right)$, except for the English in $(\mathrm{P}<0.2)$, which is close. For an in-depth discussion of the error patterns, see section 4.4 .

Table 3. Summary of correct labels for each preposition category tested

\begin{tabular}{|lll|lll|}
\hline \multicolumn{3}{|c|}{ English } & \multicolumn{3}{c|}{ Dutch } \\
\hline in & 6 & $75 \%$ & in & 6 & $75 \%$ \\
& & & op & 7 & $87 \%$ \\
on & 21 & $87 \%$ & aan & 6 & $75 \%$ \\
& & & om & 8 & $100 \%$ \\
\hline
\end{tabular}

Recall that within each generalization context, SEQL was free to make as many generalizations as it liked. SEQL was also able to keep some cases as exemplars if they did not match any of the other cases in the context. The table below summarizes the number of generalizations and exemplars for each context.

Table 4. Number of exemplars and generalizations for each generalization context

\begin{tabular}{|l|l|l|l|l|l|l|}
\hline & \multicolumn{2}{|c|}{ English } & \multicolumn{4}{c|}{ Dutch } \\
\hline & in & on & in & op & aan & om \\
\hline Generalizations & 2 & 6 & 2 & 2 & 3 & 3 \\
\hline Exemplars & 2 & 0 & 2 & 2 & 0 & 2 \\
\hline
\end{tabular}


At first the amount of variation within the contexts might seem surprising. However, since the stimuli were chosen to cover the full range of situations for each context it makes more sense. Consider the Dutch category op. The 8 sketches for this one generalization included very different situations: clingy attachment (e.g. sticker op cupboard), traditional full support (e.g. cookie op plate) and covering relationships (e.g. top op jar).

Two of the English generalizations are shown in the figures below. For each generalization the cases that were combined are listed followed by the facts and associated probabilities.

\section{Best Generalization IN}

Size: 3

(candle in bottle, cookie in bowl, marble in water)

--DEFINITE FACTS:

(rcc8-TPP figure ground)

--POSSIBLE FACTS:

33\%: (Basin ground)

33\%: (Bowl-Generic ground)

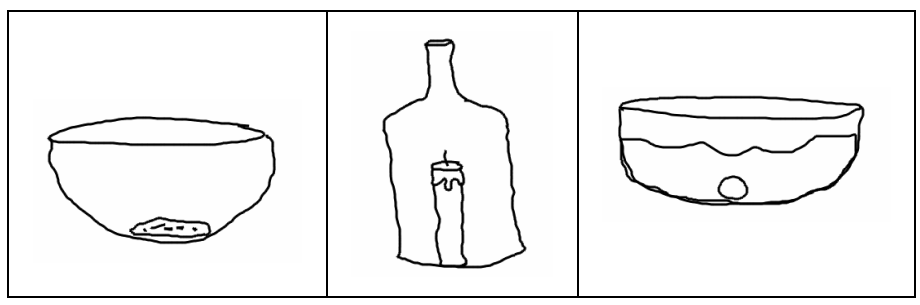

Figure 3. One of the generalizations for English in along with the sketches for the component exemplars 
Best Generalization ON

Size: 2

(top on tube, lid on jar)

--DEFINITE FACTS:

(Covering-Object figure)

(above figure ground)

--POSSIBLE FACTS:

50\%: (definiteOverlapCase figure ground)

$50 \%$ : (rcc8-PO figure ground)

$50 \%$ : (rcc8-EC figure ground)

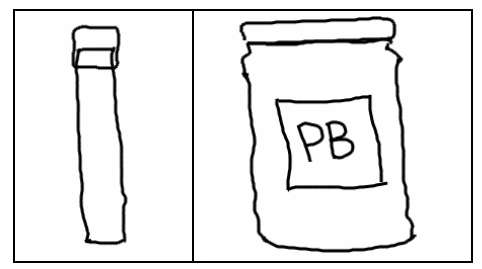

Figure 4. Sample generalizations for English on along with the component sketches

\subsection{Error Analysis}

Closer examination of the specific errors made by SEQL is also illuminating. For example, both the Dutch and English experiments failed on two in stimuli. It was the same two stimuli for both languages: flower in book, and hole in towel. The first case, flower in book, is hard to represent in a sketch. In the original study, actual objects were used making it easier to place the flower in the book. It is not surprising that this case failed given that it was an exemplar in both in contexts and did not share much structure with other stimuli in that context. Hole in towel fails for a different reason. The ResearchCyc knowledge base does not have any concept of a hole. Moreover, how holes should be considered in spatial relationships seems different than for physical objects.

Many of our errors stem from the small size of our stimuli set. For contexts that contained multiple variations, there were often only one or two samples of each. An interesting future study will be to see how many stimuli are needed to minimize error rates. (Even human adults are not $100 \%$ correct on these tasks.) Interestingly, om is one of the prepositions that is harder for Dutch children to learn (it covers situations of encirclement with support). However, it was the only Dutch preposition for which our system scored $100 \%$. This again is probably explainable by sample size. Since 
the entire context contained only cases of encirclement with support, there was more in common between all of the examples.

\subsection{Discussion}

Our results suggest that progressive alignment is a promising technique for modeling the learning of spatial language categories. Using a very small set of training stimuli (only 7 sketches in some cases) SEQL was able to correctly label the majority of the test cases. An examination of the results and errors indicates that our model, consistent with human data, uses both geometric and semantic knowledge in learning these prepositions. SEQL is able to learn these terms reasonably well, even with far less data than human children, but on the other hand, it is given very refined inputs to begin with (i.e., sketches). As noted below, we plan to explore scaling up to larger stimulus sets in future work.

\section{Related Work}

There has been considerable cognitive science research into spatial prepositions, including a number of computational models. Most computational models (cf. [16, 18, 10]) are based only on geometric information, which means that they cannot model findings of Coventry et al [6] and Feist \& Gentner[9], who showed that semantic knowledge of functional properties is also crucial. Prior computational models have also focused only on inputs consisting of simple geometric shapes (squares, circles, triangles, etc.). We believe our use of conceptually labeled sketches is an interesting and practical intermediate point between simple geometric stimuli and full 3D vision.

We also differ from many other models of spatial language use in the number of training trials required. Many current models use orders of magnitude more trials than we do. We are not arguing that people learn spatial preposition categories after exposure to only 7 examples. After all, children have a much harder task than the one we have modeled here: they have many more distractions and a much richer environment from which to extract spatial information. On the other hand, we suspect that requiring $10^{3}-10^{4}$ exposures, as current connectionist models need, is psychologically implausible. For example, one model requires an epoch of 2100 stimuli just to learn the distinction above/below/over/under for one arrangement of objects (a container pouring a liquid into a bowl/plate/dish) [7]. The actual number of trials that is both sufficient and cognitively plausible remains an open question and an interesting problem for future work.

\section{Conclusions and Future Work}

Our model was able to successfully learn the support-containment prepositions in both Dutch and English with a small number of training trials. We see three lines of 
investigation suggested by these results. First, we would like to expand our experiments to include more relationships (e.g. under, over, etc). Second, we would like to expand to other languages. For example, Korean uniquely divides the containment relationship into tight fit and loose fit relations. Third, we are in the process of building a sketch library of more instances of spatial relations. With more sketches, we will have additional evidence concerning the coverage of our model.

There is also clearly a tradeoff between using a cognitively plausible number of training examples and having enough training examples to get good generality. For example, being able to automatically extract the important object types and features (e.g. containers) and ignore the spurious ones (e.g. that something is edible). We are planning future experiments to examine this issue by varying the number of training trials used. It will also be interesting to see if we can use the same set of experiments to model the development of spatial language categories in children by varying the availability of different types of information.

Acknowledgments. This work was sponsored by a grant from the Intelligent Systems Program of the Office of Naval Research and by The National Science Foundation under grant no: SBE-0541957, The Spatial Intelligence and Learning Center. The authors would like to thank Dedre Gentner and Melissa Bowerman for access to their in-press paper and stimuli.

\section{References}

1. Bowerman, M., \& Pederson, E. Crosslinguistic perspectives on topological spatial relationships. Paper presented at the $87^{\text {th }}$ Annual Meeting of the American Anthropological Association, San Francisco, CA (1992).

2. Bowerman, M. Learning How to Structure Space for Language: A Crosslinguistic Perspective. In P. Bloom, M.A. Peterson, L.Nadel, and M.F. Garrett (eds.). Language and Space. 493-530. Cambridge, Mass. MIT Press. (1996).

3. Cohn, A. Calculi for Qualitative Spatial Reasoning. In Artificial Intelligence and Symbolic Mathematical Computation, LNCS 1138, eds: J Calmet, J A Campbell, J Prfalzgraph, Springer Verlag, 124-143. (1996).

4. Coventry, K.R., Prat-Sala, M., \& Richards, L.V. The Interplay Between Geometry and Function in the Comprehension of 'over', 'under', 'above', and 'below'. Journal of Memory and Language, 44, 376-398. (2001).

5. Coventry, K.R., \& Mather, G. The real story of 'over'?. In K.R. Coventry \& P.Oliver (Eds), Spatial Language: Cognitive and Computational Aspects. Dordrecht, The Netherlands: Kluwer Academic Publishers. (2002).

6. Coventry, K.R. \& Garrod, S.C. Saying, Seeing and Acting: The Psychological Semantics of Spatial Prepositions. Lawrence Erlbaum Associates. Essays in Cognitive Science Series. (2004).

7. Coventry, K.R., Cangelosi, A., Rajapakse, R., Bacon, A., Newstead, S., Joyce, D., and Richards, L.V. Spatial prepositions and vague quantifiers: Implementing the functional geometric framework. In Proceedings of Spatial Cognition Conference. Germany: Springer Verlag. (2005). 
8. Falkenhainer, B., Forbus, K.,\& Gentner, D. The Structure-Mapping Engine. Proceedings of the Fifth National Conference on Artificial Intelligence (pp. 272277). San Francisco, CA: Morgan Kaufmann, (1986).

9. Feist, M.I. \& Gentner, D. On Plates, Bowls, and Dishes: Factors in the Use of English 'in' and 'on'. Proceedings of the 20th Annual Conference of the Cognitive Science Society. (1998).

10. Gapp, K.P. Angle, distance, shape and their relationship to project relations. In J.D. Moore \& J.F. Lehman (Eds.), Proceedings of the Seventeenth Annual Conference of the Cognitive Science Society. (pp. 112-117). Mahwah, NJ: Lawrence Erlbaum Associates Inc. (1995).

11. Gentner, D. \& Bowerman, M. Why Some Spatial Semantic Categories are Harder to Learn than Others: The Typological Prevalence Hypothesis. (in press).

12. Gentner, D. Structure-Mapping: A theoretical framework for analogy. Cognitive Science, 7, 155-170, (1983).

13. Gentner, D., \& Markman, A. B. (1997). Structure mapping in analogy and similarity. American Psychologist, 52, 42-56.

14. Halstead, D., \& Forbus, K. Transforming between Propositions and Features: Bridging the Gap. Proceedings of AAAI, Pittsburgh, PA (2005).

15. Kuehne, S., Forbus, K., Gentner, D., \& Quinn, B. SEQL: Category learning as progressive abstraction using structure mapping. Proceedings of the 22nd Annual Meeting of the Cognitive Science Society (2000).

16. Lockwood, K., Forbus, K., Halstead, D., \& User, J. Automatic Categorization of Spatial Prepositions. Proceedings of the 28th Annual Conference of the Cognitive Science Society. (2006).

17. Markman, A. B., \& Gentner, D. (1996). Commonalities and differences in similarity comparisons. Memory \& Cognition, 24(2), 235-249.

18. Regier, T. The human semantic potential: Spatial language and constrained connectionism, Cambridge Mass: MIT Press (1996).

19. Regier, T. \& Carlson, L.A. Grounding spatial language in perception: An empirical and computational investigation. Journal of Experimental Psychology: General, 130(2), 273-298 (2001).

20. Skorstad, J., Gentner, D., \& Medin, D. Abstraction Process During Concept Learning: A Structural View. Proceedings of the 10th Annual Conference of the Cognitive Science Society. (1988). 